\title{
Comparison Study of Darker Image Edges using Min Constructor-Gaussian Operator and Traditional Operators
}

\author{
Sasmita Mishra \\ Professor, Department of CSEA, IGIT, Sarang, Odisha, India
}

\begin{abstract}
Edge detection is an important technique in image processing .In this paper, a new approach to edge detection (Min Constructor - Gaussian Operator) is presented. Here the result of some traditional edge detection methods such as Sobel,Prewitt and Robert operator are compared with this new approach.
\end{abstract}

Keywords: Edge Detection, Image processing and Min Constructor-Gaussian operator, Sobel Operator, Prewitt Operator, Robert Operator.

\section{INTRODUCTION}

Edge detection algorithm is essentially a process of detection of this discontinues in an image.The nature of intensity variation points to application of derivative operators for detecting edges.Application of derivative operator on intensity image produce another image ,usually called gradient image as it reveals the rate of intensity variation. The image is then made to undergo thresholding and /or edge linking in order to yield contours. There are many ways to perform edge detection. However, the majority of different methods may be grouped into two categories, Gradient and Laplacian. The gradient method detects the edges by looking for the maximum and minimum in the first derivative of the image. The Laplacian method searches for zero crossings in the second derivative of the image to find edges. An edge has the one-dimensional shape of a ramp and calculating the derivative of the image can highlight its location.

The goal of edge detection is to mark the points in a digital image at which the luminous intensity changes sharply. Sharp changes in image properties usually reflect important events and changes in properties of the world. These include discontinuities in depth, discontinuities in surface orientation, changes in material properties and Variations in scene illumination. Edge detection is a research field within image processing and computer vision, in particular within the area of feature extraction.

There are many methods for edge detection[1], but most of them can be grouped into two categories, search-based and zero-crossing based. The search-based methods detect edges by first computing a measure of edge strength, usually a first-order derivative expression such as the gradient magnitude, and then searching for local directional maxima of the gradient magnitude using a computed estimate of the local orientation of the edge, usually the gradient direction. The zero-crossing based methods search for zero crossings in a second-order derivative expression computed from the image in order to find edges, usually the zero-crossings of the Laplacian or the zero-crossings of a non-linear differential expression, as will be described in the section on differential edge detection following below. As a pre-processing step to edge detection, a smoothing stage, typically Gaussian smoothing[3], is almost always applied.

\section{TRADITIONAL EDGE DETECTION TECHNIQUES}

There are several traditional edge detection techniques. These techniques detect the edges by using different edge detection masks.

\section{A.SOBEL EDGE DETECTION MASK}

\begin{tabular}{|c|c|c|}
\hline-1 & 0 & +1 \\
\hline-2 & 0 & +2 \\
\hline-1 & 0 & +1 \\
\hline \multicolumn{4}{|c|}{$G_{X}$}
\end{tabular}

\begin{tabular}{|c|c|c|}
\hline+1 & +2 & +1 \\
\hline 0 & 0 & 0 \\
\hline-1 & -2 & -1 \\
\hline
\end{tabular}

Fig. 1 Sobel Operator Mask

\section{B.PREWITT EDGE DETECTION MASK}

$$
\begin{gathered}
\mathrm{G}_{X}=\left[\begin{array}{ccc}
-1 & 0 & +1 \\
-1 & 0 & +1 \\
-1 & 0 & +1
\end{array}\right] * A \\
\mathrm{G}_{Y=}\left[\begin{array}{ccc}
+1 & +1 & +1 \\
0 & 0 & 0 \\
-1 & -1 & -1
\end{array}\right] * A
\end{gathered}
$$

Fig. 2 Prewitt Operator Mask

\section{C.ROBERT EDGE DETECTION MASK}

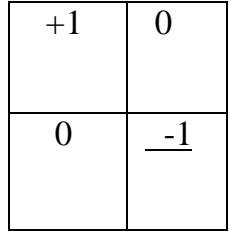

GX

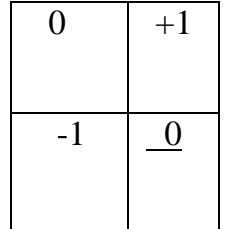

GY
Fig.3 Robert Operator Mask 


\section{MIN CONSTRUCTOR-GAUSSION EDGE DETECTION METHOD}

The min constructor is a generalization of the tnprocessing

A t-norm $\mathrm{T}:[0,1]^{2} \rightarrow[0,1]$ is an associative, commutative, increasing function, such that, $\mathrm{T}(1, \mathrm{x})=\mathrm{x}$ for all $\mathrm{x} \in[0,1]$. At-norm $\mathrm{T}$ is called idempotent if $\mathrm{T}(\mathrm{x}$, $\mathrm{x})=\mathrm{x}$ for all $\mathrm{x} \in[0,1]$.

The four basic $t$-norms are as follows

1. The minimum $\mathrm{T}_{\mathrm{M}}(\mathrm{x}, \mathrm{y})=\min (\mathrm{x}, \mathrm{y})$.

2. The product $\mathrm{T}_{\mathrm{P}}(x, y)=x \cdot y$.

3. The Łukasiewicz t-norm

$$
\mathrm{T}_{\mathrm{L}}(x, y)=\max (\mathrm{x}+\mathrm{y}-1,0) .
$$

4. The nilpotent minimum t-norm

5. $\mathrm{T}_{\mathrm{nM}}(\mathrm{x}, \mathrm{y})=\min (x, y)$, if $x+y>1$

$$
0 \text {, otherwise. }
$$

- $\quad$ Let $\mathrm{R} \in \mathrm{F}(\mathrm{X} \times \mathrm{Y})$ be an FR. Consider two tnorms $\mathrm{T} 1$ and $\mathrm{T} 2$ and two values $\mathrm{n}, \mathrm{m} \in \mathrm{N}$ so that $\mathrm{n} \leq \mathrm{P}$ $-1 / 2$, and $\mathrm{m} \leq \mathrm{Q}-1 / 2$. We define the lower constructor associated with $\mathrm{T} 1, \mathrm{~T} 2, \mathrm{n}$, and $\mathrm{m}$ in the following way:

- $\quad \mathrm{L}^{\mathrm{n}, \mathrm{m}} \mathrm{T} 1, \mathrm{~T} 2: \mathrm{F}(\mathrm{X} \times \mathrm{Y}) \rightarrow$ given by

$$
\begin{aligned}
& \mathrm{L}^{\mathrm{n}, \mathrm{m}} \mathrm{T} 1, \mathrm{~T} 2[\mathrm{R}](\mathrm{x}, \mathrm{y})= \\
& \qquad \begin{array}{c}
\mathrm{T}_{1}{ }^{\mathrm{m}, \mathrm{n}}\left(\mathrm{T}_{2}(\mathrm{R}(\mathrm{x}-\mathrm{i}, \mathrm{y}-\mathrm{j}), \mathrm{R}(\mathrm{x}, \mathrm{y}))\right) \\
\mathrm{i}=-\mathrm{n} \\
\mathrm{J}=-\mathrm{m} \\
\text { for } \quad \text { all }(\mathrm{x}, \mathrm{y}) \in(\mathrm{X}, \mathrm{Y})
\end{array}
\end{aligned}
$$

The Algorithm begins with reading an $\mathrm{M} x \mathrm{~N}$ image. The first set of nine pixels of a $3 \times 3$ window are chosen with central pixel having values $(2,2)$ i.e for each pixel $(i, j)$ we are taking the 8 neighbourhood of $(i, j)$.After the initialization, the pixel values are initially marked as edge pixel after an observation to the 8 neighbourhood. After the subjection of the pixel values the algorithm generates an intermediate image using a construction method stated below. It is checked whether all pixels have been checked or now, if not then first the horizontal coordinate pixels are checked. If all horizontal pixels have been checked the vertical pixels are checked else the horizontal pixel is incremented to retrieve the next set of pixels of a window. In this manner the window shifts and checks all the pixels in one horizontal line then increments to check the next vertical location.

After edge highlighting image is subjected to another set of condition with the help of which the unwanted parts of the output image of such type are removed to generate an image which contains only the edges associated with the input image. For an input image $\mathrm{A}$ and an output image $B$ of size $M \times N$ pixels respectively we have the following set of conditions that are implemented to detect the edges pixel values.

Input: An image $\mathrm{A}$ of $\mathrm{M} x \mathrm{~N}$ pixels (Phase 1)

Output: An image B of M x N pixels

Initial Edge Detection (A, B) using Min Construction

For $\mathrm{I} \leftarrow 2$ to $\mathrm{M}-1$

For $\mathrm{J} \leftarrow 2$ to $\mathrm{N}-1$

$$
\begin{aligned}
& \text { If A }(\mathrm{I}-1, \mathrm{~J})>\mathrm{A}(\mathrm{I}-1, \mathrm{~J}+1) \\
& \text { Then If } \mathrm{A}(\mathrm{I}-1, \mathrm{~J}-1)>\mathrm{A}(\mathrm{I}, \mathrm{J}) \\
& \text { Then If } \mathrm{A}(\mathrm{I}, \mathrm{J}-1)>\mathrm{A}(\mathrm{I}+1, \mathrm{~J}-1)
\end{aligned}
$$

$$
\begin{aligned}
& \text { Then } \\
& \text { B (I-1, J+1) Ł0 } \\
& \mathrm{B}(\mathrm{I}, \mathrm{J}) \longleftarrow 0 \\
& \mathrm{~B}(\mathrm{I}+1, \mathrm{~J}-1) \leftarrow 0
\end{aligned}
$$

End For

End For

For $\mathrm{I} \leftarrow 2$ to $\mathrm{M}-1$

For $\mathrm{J} \leftarrow 2$ to $\mathrm{N}-1$ $1)=255$

If $\mathrm{B}(\mathrm{I}-1, \mathrm{~J})=255 \& \quad \mathrm{~B}(\mathrm{I}, \mathrm{J})=0 \& \quad \mathrm{~B}(\mathrm{I}+1, \mathrm{~J})=255 \& \quad \mathrm{~B}(\mathrm{I}, \mathrm{J}-$ initially.

Then B (I, J) is minimum and highlighted as edge

End For

End For

In the above algorithm Min construction[3] is used but not after fuzzification as after fuzzification the membership values would become fractions that can't be stored in unsigned char. Hence the same technique of min construction is used but on true picture and taking into consideration 8-nbd of a pixel (i,j).

We can observe in the above algorithm written for a particular fuzzy condition that the nesting of statements is done in a manner that only the edge associated pixels are granted black pixel values and initially min valued edge pixels are given white value. These pixels are initially marked as edge.

Phase 2. Input: An image $\mathrm{B}$ of size $\mathrm{MxN}$ Output: Edge image of size $\mathrm{MxN}$

We now use Gaussian operator[11] on the intermediate image to get the edge image. And In this way whatever image is being constructed is compared with edges found on same image by other existing techniques.

GAUSSION operator mask is given as:

$$
\left[\begin{array}{ccccc}
0 & 0 & 1 & 0 & 0 \\
0 & 1 & 2 & 1 & 0 \\
1 & 2 & -16 & 2 & 1 \\
0 & 1 & 2 & 1 & 0 \\
0 & 0 & 1 & 0 & 0
\end{array}\right]
$$

Fig. 4 Gaussian Operator Mask

\section{EXPEIMENTAL RESULTS}

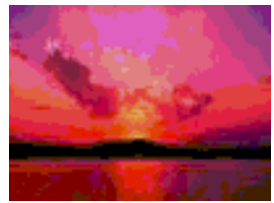

Fig. 5 Original Image

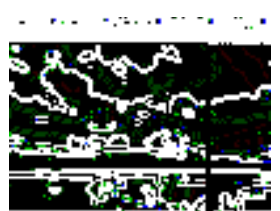

Fig. 6 Result Using Sobel Operator Mask 


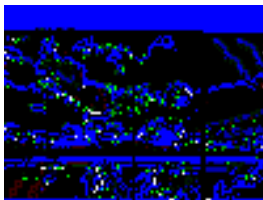

Fig. 7 Result Using Prewitt Operator Mask

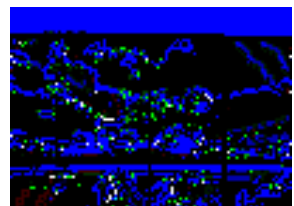

Fig. 8 Result Using Robet Operator Mask

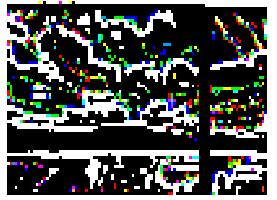

Fig. 9 Result Using Min Constructor-Gaussian Edge Detection Mask

\section{CONCLUSION}

In this paper, Comparison were made amongst the various edge detection algorithms with the purposed method. From the result analysis, it is concluded that the new concept Min Constructor-Gaussion operator provides better result for darker images compared to the traditional operators

\section{REFERENCES}

[1] H. Bustince,E. Barrenechea, M. Pagola and R. Orduna "Construction of interval type-2 fuzzy images to represent images in gray scale:false edges ," in Proc. IEEE Int. Conf. Fuzzy Syst. London, U.K., PP. 73-78,2007.

[2] Mitra Basu, Senior Member, IEEE, "Gaussian-Based EdgeDetection Methods," IEEE Transactions On Systems, Man, And Cybernetics-Part C: Applications And Reviews, Vol. 32, No. 3 , August 2008.

[3] Edurne Barrenechea, Humberto Bustince, Member, IEEE, Bernard De Baets, And Carlos Lopez-Molina, Student Member, IEEE, "Construction Of Interval-Valued Fuzzy Relations With Application To The Generation Of Fuzzy Edge Images," IEEE Transactions On Fuzzy Systems, Vol. 19, No. 5, pp. 819-830, October 2011

[4] H. Bustince,E. Barrenechea, M. Pagola and J. Fernandez, "Interval valued fuzzy sets constructed from matrices:Application to edge [

[5] Zhao Yu-qian, Gui Wei-hua, Chen Zhen-cheng, Tang Jing- tian and Li Ling-yun,"Medical Images Edge Detection Based on [.

[6] Hui LI , Pengfeng XIAO , Xuezhi FENG , Chunjing WEN , Chongya JIANG"Multiscale Feature Detection of Multispectral Remotely Sensed Imagery in Wavelet Domain," IEEE, 2009.

[7] Sankar Krishnamurthy,"Histogram based Morphological Edge Detector," ,IEEE Transaction on Geosciences and Remote Sensing , Vol.32, No.4, July 1994.

[8] Morris o.;M.Lee," A unified method for segmentation \& edge detection using graph theory," Acoustics ,Speech \& Signal processing ,IEEE international conference,ICASSP'86,Vol.11, pp2051-2054, Apr.1986.

[9] P.J.Symonds,"A new technique for automatically segmenting images of the surface froth structures thatare prevalent in industrial flotation cells," IEEE transaction, 1992.

[10] Stamatia Giannarou and Ania Stataki., "Edge Detection Using Quantitative Combination," IEEE transactions on signal [.

[11] M. Basu, " Gaussian-based edge-detection methods: A survey ,' IEEE Trans. Syst. ,Man, Cybern. C, Appl. Rev., vol. 32, no. 3, pp. 252-260, Aug.2002

[12] G. Deschrijver, C. Cornelis and E.E. Kerre, "On the representation of intuitionistic fuzzy t-norms and t-conorms ," IEEE Trans. Fuzzy Syst., vol.12, No.1, pp.45-61, Feb.2004.
[13] J.M Mendel,R.I. John and F. Liu, "Interval type-2 fuzzy logic systems made simple ," IEEE Trans. Fuzzy Syst. ,vol. 14,No. 6, pp. 808-821, Dec. 2006.

[14] R. Medina-Carnicer,A. Carmona-Poyato,R. Munoz-Salinas and F. J. Madrid-Cuevas, "Determining hysteresis thresholds for edge detection by combining the advantages and disadvantages of thresholding methods ," IEEE Trans. Image Process. , Vol. 19, No. 1 , pp. 165-173, Jan. 2010.

[15] Q. Liang and J.M. Mendel, "Interval type-2 fuzzy logic systems:Theory and design ," IEEE Trans. Fuzzy Syst., Vol. 8, No. 5, pp. 535-550, Oct. 2000. 